\title{
2019 Canadian Urological Association (CUA)-Canadian Uro Oncology Group (CUOG) guidelines for the management of castration-resistant prostate cancer (CRPC)
}

Fred Saad ${ }^{1}$; Armen Aprikian²; Antonio Finelli ${ }^{3}$; Neil E. Fleshner ${ }^{3}$; Martin Gleave ${ }^{4}$; Anil Kapoor $^{5}$; Tamim Niazi ${ }^{6}$; Scott North ${ }^{7}$; Frederic Pouliot ${ }^{8}$; Ricardo Rendon ${ }^{9}$; Bobby Shayegan ${ }^{5}$; Srikala Sridhar ${ }^{10}$; Alan So ${ }^{4}$; Nawaid Usmani ${ }^{11}$; Eric Vigneault ${ }^{12}$; Kim N. Chi ${ }^{13}$ ${ }^{1}$ Centre Hospitalier de l'Université de Montréal, Montreal, QC, Canada; ${ }^{2}$ McGill University Health Center, Montreal, QC, Canada; ${ }^{3}$ Division of Urology, University of Toronto, Toronto, ON, Canada; ${ }^{4}$ Deptartment of Urological Sciences, University of British Columbia, Vancouver, BC, Canada; ${ }^{5}$ Department of Surgery (Urology) and Oncology, McMaster University, Hamilton, ON, Canada; ${ }^{6}$ Jewish General Hospital, Montreal, QC, Canada; ${ }^{7}$ Department of Medical Oncology, University of Alberta, Edmonton, AB, Canada; ${ }^{8} \mathrm{CHU}$ de Quebec, Université Laval, Quebec City, QC, Canada; ${ }^{9}$ Department of Urology, Dalhousie University, Halifax, NS, Canada; ${ }^{10}$ Division of Medical Oncology, University of Toronto, Toronto, ON, Canada; ${ }^{11}$ Department of Oncology, Division of Radiation Oncology, University of Alberta, Edmonton, AB, Canada; ${ }^{12}$ Department of Radiation Oncology, CHU de Quebec, Université Laval, Quebec City, QC, Canada; ${ }^{13}$ BC Cancer Agency, Vancouver, BC, Canada

Cite as: Can Urol Assoc J 2019 June 25; Epub ahead of print. http://dx.doi.org/10.5489/cuaj.6136

Published online June 25, 2019

$* * *$

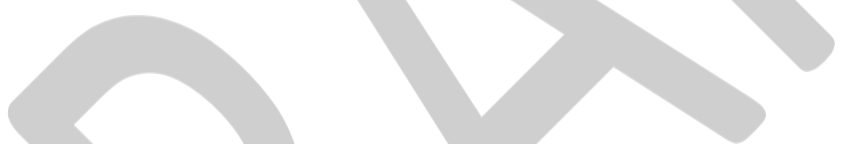

MEDLINE search of the English language and conference proceedings were used to produce the present document. Wherever Level 1 evidence is lacking, the guideline attempts to provide expert opinion to aid in the management of patients.

Levels of evidence and grades of recommendation employ the International Consultation on Urologic Disease (ICUD)/WHO modified Oxford Center for Evidence-Based Medicine grading system. Based on a modified GRADE methodology, the strength of each recommendation is represented by the words STRONG or WEAK.

\section{Introduction}

Castration-resistant prostate cancer (CRPC) is defined by disease progression despite castrate levels of testosterone and may present as either a continuous rise in serum prostate-specific antigen (PSA) levels, the progression of pre-existing disease, and/or the appearance of new metastases.

Advanced prostate cancer has been known under a few names over the years, including hormone-resistant prostate cancer (HRPC) and androgen-insensitive prostate cancer (AIPC). Most recently, the terms castration-resistant prostate cancer or castration-recurrent prostate 
cancer were introduced with the realization that extra-testicular androgen production plays a significant role in the resistance of prostate cancer cells to medical or surgical castration therapy. ${ }^{1}$

In their second publication, the Prostate Cancer Working Group defined CRPC as a continuum on the basis of whether metastases are detectable (clinically or by imaging) and whether the serum testosterone is in the castrate range by surgical orchidectomy or medical therapy. ${ }^{2}$ This definition creates a clinical-states model, where patients can be classified. The rising prostate-specific antigen (PSA) states (castrate and non-castrate) signify that no detectable (measurable or non-measurable) disease has ever been found. The clinical metastases states (castrate and non-castrate) signify that disease was detectable at some point in the past, regardless of whether it is detectable now. ${ }^{3}$

Prognosis is associated with several factors that go beyond PSA levels. These include performance status, presence of visceral metastases, presence of bone pain, extent of disease on bone scan, and serum lactate dehydrogenase and alkaline phosphatase levels. Bone metastases will occur in $90 \%$ of men with CRPC and can produce significant morbidity, including pain, pathologic fractures, spinal cord compression, and bone marrow failure. Paraneoplastic effects, including anemia, weight loss, fatigue, hypercoagulability, and increased susceptibility to infection, are also common.

CRPC includes patients without metastases or symptoms with rising PSA levels despite androgen-deprivation therapy (ADT) to patients with metastases and significant debilitation due to cancer symptoms.

\section{Management of CRPC}

Androgen-deprivation therapy and first-generation androgen receptor antagonists

Because the androgen receptor remains active in most patients who have developed castration-resistant disease, it is recommended that ADT be continued for the remainder of a patient's life (Level 3, Weak Recommendation). In patients who develop CRPC, the addition or change of first-generation androgen receptor antagonists may be considered (Level 3, Weak Recommendation).

To date, no study using first -generation androgen receptor antagonists, when introduced in the CRPC setting, has shown survival benefits; most trials have been small, were not designed to evaluate overall survival (OS), and were heavily confounded by future treatments used. In patients treated with luteinizing hormone-releasing hormone (LHRH) agonist/antagonist monotherapy or those who have had an orchidectomy, the addition of androgen receptor antagonists, such as bicalutamide, can offer modest PSA responses that are short-lived in 30$35 \%$ of patients. ${ }^{4}$

For patients who have undergone TAB, the anti-androgen (AA) should be discontinued to test for an anti-androgen withdrawal response (AAWD). Changing AA or using corticosteroids 
with or without ketoconazole have been noted to cause transient PSA reductions in about 30\% of patients but have not been shown to improve any of the clinically meaningful outcome measures.

\section{Non-metastatic CRPC (nmCRPC)}

For men with high-risk nmCRPC, defined as a PSA doubling time (PSADT) of less than 10 months, with an estimated life expectancy of greater than five years should be offered apalutamide or enzalutamide (Level 1, Strong Recommendation).

Until 2018, there was no standard of care and no approved regimen for the nmCRPC state. The risk of progression to clinical metastases or death is linked to PSADT. PSADT of less than 10 months has been correlated to worse outcome and has been used in recent clinical trials as the definition for high-risk nmCRPC. Patients in these studies were randomized to treatment + ADT vs. placebo + ADT until the appearance of metastases on conventional imaging (bone scan and computed tomography [CT]/magnetic resonance imaging [MRI] of the abdomen/chest). To date, two phase 3 studies have led to Health Canada approvals for the treatment of nmCRPC in Canada. Both studies used second-generation androgen receptor (AR)-targeted therapies (apalutamide and enzalutamide) and reported similar results in significantly improving the primary endpoint of metastases-free survival (MFS). Median OS, a secondary endpoint, was not reached but at interim analysis showed a non-significant improvement in both studies(5,6). Recently darolutamide was tested in a similar patient population of high risk nmCRPC with positive results. At this time this agent is not approved in Canada. (ARAMIS) (7)

\section{Summary of results}

Apalutamide

Apalutamide is a second-generation AR ligand-binding domain inhibitor. This agent was tested in combination with standard ADT in patients with nmCRPC at high risk for progression (PSADT of $\leq 10$ months)(5). The median MFS was 40.5 months with apalutamide and 16.2 months with placebo (hazard ratio [HR] for metastasis or death 0.28 ; 95\% confidence interval [CI] 0.23-0.35; $\mathrm{p}<0.001)$. Secondary endpoints analyzed, including progression-free survival (PFS) (local and distant), time to PSA progression, and time to subsequent therapy, were all statistically significantly improved(5). Although more adverse events were reported in patients receiving ADT + apalutamide vs. ADT + placebo, patient-reported health-related quality of life was similar between both groups. (8)

\section{Enzalutamide}

Enzalutamide is a second-generation AR ligand-binding domain inhibitor. This agent was tested in combination with standard ADT in patients with nmCRPC at high risk for progression (PSADT of $\leq 10$ months)(6). The median MFS was 36.6 months with enzalutamide and 14.7 months with placebo (HR for metastasis or death 0.29; 95\% CI 0.24-0.35; p<0.001). Secondary endpoints analyzed, including PFS (local and distant), time to PSA progression, and time to subsequent therapy, were all statistically significantly improved. Although more adverse events 
were reported in patients receiving ADT + enzalutamide vs. ADT + placebo, patient-reported health-related quality of life was similar between both groups. (9)

Both apalutamide and enzalutamide have received Health Canada approval for use in high-risk non-metastatic CRPC.

\section{Detection of metastases and imaging in untreated patients}

For patients who progress on ADT without evidence of distant metastases, it is suggested to screen for bone metastases with bone scans and monitor for lymph node and visceral metastases/progression with imaging of the abdomen/pelvis and chest.

Patients with a rapid PSADT ( $<10$ months) or elevated PSA levels $(>20)$ are at high risk for developing metastases earlier (3). Imaging in these patients should be performed every 3-6 months. Patients with a slower PSADT (>10 months) should be screened every 6-12 months (Expert opinion). Imaging techniques most commonly used include nuclear bone scans and abdominal/pelvic CT and chest X-ray. The role of positron-emission tomography (PET) such as PSMA-PET are still unclear and the benefits unknown.

\section{Guideline statements regarding nmCRPC}

1. ADT should be maintained in the nmCRPC state. First-generation androgen receptor antagonists (i.e., bicalutamide, flutamide, etc.) should be discontinued if patients are receiving these agents (Level 3, Weak Recommendation).

2. For men with high-risk nmCRPC, defined as a PSADT $<10$ months, with an estimated life expectancy of greater than five years should be offered apalutamide or enzalutamide (Level 1, Strong Recommendation).

3. In men with high-risk nmCRPC who are felt to be unsuitable or refuse approved therapies, observation or use of first-generation androgen receptor antagonists may be attempted (Level 3, Weak Recommendation).

4. Men with nmCRPC who are not considered high-risk, observation or secondary hormonal treatments may be attempted (Level 3, Weak Recommendation).

5. Patients who are untreated for nmCRPC should be followed with regular imaging every 6-12 months depending on PSADT (Level 3, Weak Recommendation).

If and when metastases are detected, patients should be treated according to guidelines for mCRPC. How patients are treated in the mCRPC state will depend on what they received prior to becoming mCRPC.

Treatment of metastatic CRPC (mCRPC)

Since mCRPC is generally associated with a high risk of morbidity and cancer-related mortality, patients with mCRPC detected on conventional imaging should be considered for systemic therapy with demonstrated survival benefits. Patients with mCRPC should optimally receive multidisciplinary care to maximize survival and quality of life. Because 
any treatment for advanced disease remains non-curative, patients with advanced prostate cancer should be encouraged to participate in clinical trials.

\section{AR signaling therapeutic options}

In men with CRPC, phase 3 clinical trials have evaluated the role of abiraterone acetate and enzalutamide in both the chemo-naive and post-chemotherapy settings.

\section{Abiraterone acetate}

Abiraterone acetate is a potent and irreversible inhibitor of CYP-17, a critical enzyme in androgen biosynthesis.

In the chemo-naive setting: Abiraterone acetate $1000 \mathrm{mg} / \mathrm{day}$ plus prednisone $5 \mathrm{mg}$ twice daily is recommended for first-line therapy for asymptomatic or minimally symptomatic metastatic CRPC (Level 1, Strong Recommendation).

In asymptomatic or minimally symptomatic patients (defined as pain that is relieved by acetaminophen or a non-steroidal anti-inflammatory) without visceral metastases, abiraterone acetate significantly improved radiographic PFS (16.5 vs. 8.3 months) (HR 0.53; 95\% CI 0.45$0.62 ; \mathrm{p}<0.001)$. and had a statistically significant 4.4 months improvement in overall survival (HR 0.81; $\mathrm{p}=0.0033)(10,11)$. Abiraterone also significantly delayed time to pain progression, time to chemotherapy initiation, time to opiate initiation, and deterioration of the Eastern Cooperative Oncology Group (ECOG) performance status.

In the post-docetaxel setting: Abiraterone acetate $1000 \mathrm{mg}$ per day plus prednisone 5 mg twice daily is recommended in patients progressing on or after docetaxel-based chemotherapy (Level 1, Strong Recommendation).

In the post-docetaxel setting, abiraterone-prednisone compared to placebo-prednisone significantly prolonged median overall survival by 4.6 months (15.8 vs. 11.2 months; HR 0.74; $\mathrm{p}=0.0001$ ) in patients with mCRPC who had progressed after docetaxel treatment. Moreover, all secondary endpoints provided support for the superiority of abiraterone over placebo; median time to PSA progression (8.5 vs. 6.6 months; HR 0.63; p<0.0001), radiographic PFS (5.6 vs. 3.6 months; HR 0.66; $\mathrm{p}<0.0001$ ), confirmed PSA response rate defined as $\geq 50 \%$ reduction in PSA from the pretreatment baseline PSA (29\% vs. 5.5\%; $\mathrm{p}<0.0001$ ), and objective response by Response Evaluation Criteria in Solid Tumours (RECIST) $(14.8 \%$ vs. 3.3\%; $\mathrm{p}<0.0001) .{ }^{12}$

\section{Enzalutamide}

Enzalutamide is a potent multi-targeted androgen signalling pathway inhibitor.

In the chemo-naive setting: Enzalutamide $160 \mathrm{mg}$ per day is recommended as firstline therapy for asymptomatic or minimally symptomatic mCRPC (Level 1, Strong Recommendation).

In asymptomatic or minimally symptomatic patients (defined as pain that is relieved by acetaminophen or a non-steroidal anti-inflammatory), enzalutamide decreased the risk of radiographic progression or death by $81 \%$ (HR 0.19; 95\% CI 0.15-0.23; $<<0.001$ ) and the risk of 
death by 29\% (HR 0.71; 95\% CI 0.60-0.84; p<0.001) as compared with placebo. The benefit of enzalutamide was demonstrated for all secondary endpoints, including time to initiation of cytotoxic chemotherapy, time to first skeletal-related event, best overall soft tissue response (59\% vs. 5\%; $\mathrm{p}<0.001$ ), time to PSA progression (HR 0.17; $<<0.001$ ), and $\geq 50 \%$ PSA decline rate $(78 \%$ vs. $4 \%$; $<0.001)$. Enzalutamide also significantly delayed time to pain progression, time to opiate initiation, and deterioration of the ECOG performance status. ${ }^{13,14}$

In the post-docetaxel setting: Enzaluatmide $160 \mathrm{mg}$ per day is recommended in patients progressing on or after docetaxel-based chemotherapy (Level 1, Strong Recommendation).

In patients previously treated with docetaxel, the trial compared enzalutamide and placebo. The study demonstrated a significant advantage in OS of 4.8 months (18.4 vs. 13.6 months; HR 0.62; $\mathrm{p}<0.0001$ ) and all secondary endpoints, including confirmed PSA response rate ( $54 \%$ vs. $2 \%$; $<<0.001$ ), soft-tissue response rate ( $29 \%$ vs. $4 \% ; \mathrm{p}<0.001$ ), time to PSA progression (8.3 vs. 3.0 months; HR 0.25; $\mathrm{p}<0.001$ ), radiographic PFS (8.3 vs. 2.9 months; HR $0.40 ; \mathrm{p}<0.001$ ), and the time to the first skeletal-related event (SRE) (16.7 vs. 13.3 months; HR $0.69 ; \mathrm{p}<0.001)$. (15)

NOTE: The studies in the chemo-naive setting did not include patients with moderate or severe symptoms; however, abiraterone and enzalutamide may be potential therapeutic options in patients who are deemed chemotherapy-ineligible or refuse chemotherapy (Expert opinion).

\section{Chemotherapy}

First-line systemic chemotherapy

Docetaxel

Docetaxel $75 \mathrm{mg} / \mathrm{m}^{2}$ intravenous (IV) every three weeks with $5 \mathrm{mg}$ oral prednisone twice daily is recommended for patients with mCRPC (Level 1, Strong Recommendation).

The TAX-327 study randomized 1006 patients to one of three treatment arms: 1) docetaxel $75 \mathrm{mg} / \mathrm{m}^{2}$ IV every three weeks; 2) docetaxel $30 \mathrm{mg} / \mathrm{m}^{2}$, weekly for five of six weeks; or 3) control therapy with mitoxantrone. ${ }^{16}$ The study reported improved survival with docetaxel (every three weeks) compared with mitoxantrone-prednisone (median survival 18.9 vs. 16.5 months; HR 0.76; 95\% CI 0.62-0.94; two-sided p=0.009). No OS benefit was observed with docetaxel given on a weekly schedule (HR 0.91; 95\% CI 0.75-1.11; two-sided p=0.36). Significantly more patients treated with docetaxel (every three weeks) achieved a pain response compared with patients receiving mitoxantrone (35\% vs. 22\%; $\mathrm{p}=0.01$ ). Quality of life response, defined as a sustained 16-point or greater improvement from baseline on two consecutive measurements, was higher with docetaxel given every three weeks ( $22 \%$ vs. $13 \%$; $p=0.009)$ or weekly ( $23 \%$ vs. $13 \%$; $p=0.005$ ) compared with mitoxantrone. PSA response rates were also statistically significantly higher with docetaxel compared to mitoxantrone.(16) 
Although patients received up to 10 cycles of treatment if no progression and no prohibitive toxicities were noted, the duration of therapy should be based on the assessment of benefit and toxicities. Rising PSA only should not be used as the sole criteria for progression; assessment of response should incorporate clinical and radiographic criteria.

Alternative therapies that have not demonstrated improvement in OS but can provide disease control, palliation, and improve quality of life include weekly docetaxel plus prednisone, and mitoxantrone plus prednisone (Level 2, Weak Recommendation).

The timing of docetaxel therapy in men with evidence of metastases but without symptoms should be discussed with patients and therapy should be individualized based on patients' clinical status and preferences (Level 3, Weak Recommendation).

Patients who do not respond to first-line ADT or who progress clinically or radiologically without significant PSA elevations may have neuroendocrine differentiation. Biopsy of accessible lesions should be considered to identify these patients; these patients should then be treated with combination chemotherapy, such as cisplatin/etoposide or carboplatin/etoposide (Level 3, Weak Recommendation).

Second-line systemic chemotherapy

Cabazitaxel

Cabazitaxel is recommended for $\mathrm{mCRPC}$ patients progressing on or following docetaxel (Level 1, Strong Recommendation).

A phase 3 study comparing cabazitaxel to mitoxantrone in patients previously treated with docetaxel has shown a statistically significant survival advantage. ${ }^{17}$ This randomized, placebo-controlled trial recruited 755 docetaxel-pretreated CRPC patients. OS was the primary endpoint of the study. Patients were randomized to receive prednisone $10 \mathrm{mg} /$ day with three times weekly mitoxantrone $12 \mathrm{mg} / \mathrm{m}^{2}$ or cabazitaxel $25 \mathrm{mg} / \mathrm{m}^{2}$. An advantage in survival emerged in favour of the cabazitaxel group, with a median survival of 15.1 months compared with 12.7 months in the mitoxantrone group (HR 0.70; 95\% CI 0.59, 0.83; $\mathrm{p}<0.0001)$.(17)

A recent phase 3 study comparing cabazitaxel $25 \mathrm{mg} / \mathrm{m}^{2}$ vs. $20 \mathrm{mg} / \mathrm{m}^{2}$ resulted in noninferiority for cabazitaxel $20 \mathrm{mg} / \mathrm{m}^{2}$ with less adverse events. Of note, in the subgroup analysis of patients who had received both docetaxel and abiraterone/enzalutamide, results appeared to favour a higher dose of cabazitaxel. (18)

\section{Other options}

For patients who have had a good response to first-line docetaxel, re-treatment with docetaxel can be considered (Expert opinion, Weak Recommendation).(19,20)

Mitoxantrone may be considered a therapeutic option in symptomatic patients with mCRPC in the first- or second-line setting. Mitoxantrone has not shown any survival advantage but may provide symptomatic relief. 


\section{Bone-targeted therapy}

\section{Life-prolonging therapy}

$\underline{\text { Radium-223 }}$

Radium-223 every four weeks for six cycles is recommended in patients with pain due to bone metastases and who do not have visceral metastases (Level 1, Strong Recommendation).

Radium-223 (previously known as alpharadin) is an intravenous alpha-emitting agent that mimics calcium, preferentially targeting bone metastases. In a randomized, phase 3 study, radium-223 given every four weeks for six cycles was compared to placebo. ${ }^{17}$ Radium-223 demonstrated a significant improvement in OS and symptomatic SREs. OS was improved by 3.6 months (HR 0.7; $<<0.0001)$ and symptomatic SREs were delayed by 5.8 months $(\mathrm{p}<0.0001)$. The study included patients with symptomatic bone metastases who were post-docetaxel or ineligible for docetaxel (21). The study excluded patients with visceral metastases or lymph node metastases greater than $3 \mathrm{~cm}$. PSA measurements while receiving radium-223 cannot provide evidence of whether patients are benefitting or not. Given the mechanism of action of the drug, alkaline phosphatase appears to be better marker of activity. A phase 3 study in the first-line mCRPC setting compared radium-223 in combination with abiraterone/prednisone vs. abiraterone/prednisone alone and demonstrated no advantage and an increased risk of fractures (22).

Radium-223 should not be combined with abiraterone and a bone-supportive agent (denosumab or zoledronic acid) should always be used when using radium-223 (Level 1, Strong Recommendation).

\section{$\underline{\text { Supportive agents }}$}

\section{Denosumab and zoledronic acid}

In men with CRPC and bone metastases, denosumab (120 mg subcutaneous [SC]) or zoledronic acid (4 mg IV) every four weeks are recommended to prevent disease-related SREs, including pathological fractures, spinal cord compression, surgery, or radiation therapy to bone (Level 1, Strong Recommendation).

Bone loss associated with ADT has been shown to increase the risk of fracture. ${ }^{23-27}$ Moreover, about $90 \%$ of patients with mCRPC will develop bone metastases, which cause local decreases in bone integrity. Patients are at significant risk of SREs that include pathological fractures, debilitating bone pain requiring palliative radiation therapy, and spinal cord compression. Quality of life is affected by these complications.

Zoledronic acid is a third-generation nitrogen containing bisphosphonate. Bisphosphonates other than zoledronic acid are not known to be effective to prevent disease- 
related SREs. In the placebo-controlled zoledronic acid study, fewer men receiving zoledronic acid had SREs (38\% vs. 49\%; $\mathrm{p}=0.02$ ) ${ }^{28}$ Zoledronic acid also increased the median time to first SRE (488 vs. 321 days; $\mathrm{p}=0.01$ ). There was an overall 36\% reduction in the rate of SREs in treated patients(28). Treatment with zoledronic acid should not be used in men with baseline creatinine clearance $<30 \mathrm{~mL} / \mathrm{min}$.

Denosumab is a fully humanized monoclonal antibody against RANK ligand. It has been shown to be effective in preventing bone loss and new vertebral fractures due to ADT. ${ }^{27}$ In the setting of mCRPC, denosumab (120 mg SC every four weeks) compared to zoledronic acid (4 mg IV every four weeks) has shown significant improvement in the time to the first SRE (20.7 vs. 17.1 months; $\mathrm{p}<0.001$ for non-inferiority; $\mathrm{p}=0.008$ for superiority), while OS and PFS were not different. ${ }^{29}$

No dose modification for renal function is necessary in the case of denosumab; however, the risk of hypocalcaemia is increased and calcium monitoring and supplementation (with calcium and vitamin D) is recommended for both denosumab and zoledronic acid. Denosumab has not been studied, however, in patients with severe renal impairment (glomerular filtration rate $<30 \mathrm{ml} / \mathrm{min}$ ).

Good oral hygiene, baseline dental evaluation for high-risk individuals, and avoidance of invasive dental surgery during therapy are recommended to reduce risk of osteonecrosis of the jaw (ONJ) for patients treated with bone-targeted therapies (Level 3, Grade C). Zoledronic acid and denosumab have been used in combination with all the agents presently in use for the treatment of mCRPC. To date, there have been no additional safety issues of concern that have been reported.

The optimal duration of zoledronic acid and denosumab in men with CRPC and bone metastases is undefined. The risk of ONJ appears to be related to time on bonetargeted therapy, therefore, caution should be taken in using these agents beyond two years.

Denosumab and zoledronic acid are not approved and not indicated for SRE prevention in the treatment of metastatic castration-sensitive prostate cancer or for bone metastases prevention.

IV. Other supportive care therapies

Systemic corticosteroid therapy

Corticosteroid therapy with low-dose prednisone or dexamethasone may also offer improvements in PSA values and/or palliative outcomes in up to $30 \%$ of patients in both symptomatic and asymptomatic men. Steroids may also exert an anti-neoplastic effect on prostate cancer (Level 3, Weak Recommendation). ${ }^{31}$ 


\section{$\underline{\text { Palliative radiation }}$}

Bone metastases from prostate cancer are often radiosensitive and most men will experience partial or complete pain relief from external beam radiation to a specific lesion (33). Studies have shown that a single fraction of standard palliative radiotherapy (RT) is as effective as five or more fractions in providing palliation. However, more patients require retreatment for pain recurrence with single fraction radiation. Stereotactic body RT (SBRT) is a more precise and may be a more effective form of palliation delivered in five or fewer treatments and may also be considered particularly for oligometastatic disease where high dose radiotherapy is currently being studied for improved oncological outcomes.

\section{Malignant spinal cord compression is an oncological emergency that requires} immediate diagnosis, if suspected, with an MRI. Options for treatment are debulking surgery + RT, vertebrectomy with stabilization and RT, or RT + steroids (Level 1, Strong Recommendation).

\section{Conclusions}

Advances in treatment for men with CRPC have improved survival and quality of life, but most, if not all, patients eventually succumb to their disease and better treatments are required. Several new agents are being studied in all states of CRPC and an increase in options is likely in the near future. Because CRPC remains an incurable and ultimately fatal illness, inclusion of patients in clinical trials remains paramount.

\section{Summary}

Health Canada-approved agents that have shown improvements in survival in mCRPC now include abiraterone, enzalutamide, docetaxel, cabazitaxel, and radium-223. Health Canadaapproved agents that have shown improvements in delaying metastases in high-risk nmCRPC include apalutamide and enzalutamide. Bone supportive agents and palliative radiation continue to play an important role in the overall management of mCRPC. Given the complexity, variety and importance of optimizing the use of these agents, a multidisciplinary team approach is highly recommended.

Conflicts of Interest: Dr. Saad has been an advisory board member for and has received payment/honoraria from Abbvie, Amgen, Astellas, Bayer, Janssen, and Sanofi; and has participated in clinical trials supported by Amgen, Astellas, Bayer, Janssen, and Sanofi. Dr. Finelli has been an advisory board member for Abbvie, Astellas, Bayer, Janssen, Ipsen, Sanofi, and TerSera; and has participated in clinical trials supported by Astellas, Bayer, and Janssen. Dr. Kapoor has been an advisory board member for BMS, Eisai, Ipsen, Merck, Novartis, Pfizer, and Roche; a speakers' bureau member for Eisai, Ipsen, Novartis, and Roche; and has received grants/honoraria from BMS, Eisai, Ipsen, Merck, Novartis, Pfizer, and Roche. Dr. Niazi has received research grants and honoraria from Abbvie, Amgen, Astellas, Astra Zeneca, Bayer, Janssen, and Sanofi; and has participated in clinical trials supported by Astellas, Ferring, Janssen, and Sanofi. Dr. North has been an advisory board member for Astellas, has received honoraria from Astra Zeneca, Astellas, Janssen, Merck, Roche, and Sanofi; and has participated in clinical trials supported by Astra Zeneca, Merck, Roche, and Sanofi. Dr. Rendon has been an advisory board and speakers' bureau 
member for and has received honoraria from Abbvie, Amgen, Astellas, Astra Zeneca, Bayer, Ferring, Jansen, and Sanofi. Dr. Shayegan has been an advisory board member for Astellas, Bayer, and Janssen; and has received a research grant from Janssen. Dr. So has been an advisory board member for Abbvie, Amgen, Astellas, Bayer, Janssen, Ferring, and TerSera; and has participated in clinical trials supported by Astellas, Ferring, and Janssen. Dr. Usmani has been an advisory board member for Amgen, Astellas, and Bayer; and has received grants from Best Medical and Concure Oncology Canada. Dr. Vigneault has been an advisory board member for Abbvie, Bayers, Ferring, and Sanofi. Dr. Chi has received honoraria from Astellas, Bayer, Janssen, and Sanofi; and has participated in clinical trials supported by Astellas, Astra Zeneca, Bayer, Eli Lilly, Essa, Janssen, Merck, Novartis, Pfizer, Roche, and Sanofi.

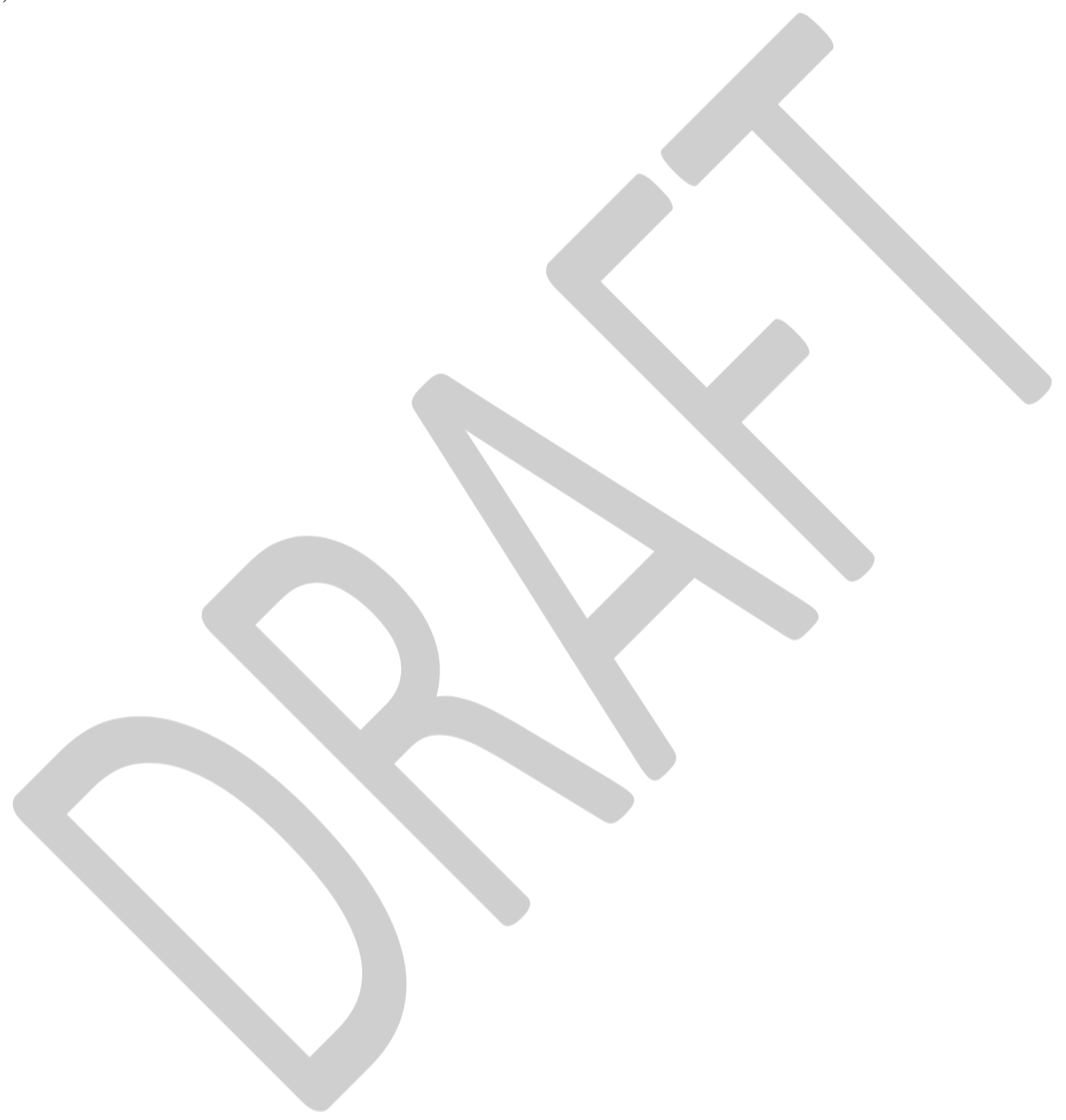




\section{References}

1. Mohler JL, Gregory CW, Ford OH, 3rd, et al. The androgen axis in recurrent prostate cancer. Clin Cancer Res 2004;10:440-8. http://dx.doi.org/10.1158/1078-0432.CCR-114603

2. Scher HI, Halabi S, Tannock I, et al. Design and end points of clinical trials for patients with progressive prostate cancer and castrate levels of testosterone: recommendations of the Prostate Cancer Clinical Trials Working Group. J Clin Oncol 2008;26:1148-59. http://dx.doi.org/10.1200/JCO.2007.12.4487

3. Smith MR, Kabbinavar F, Saad F, et al. Natural history of rising serum prostate-specific antigen in men with castrate non-metastatic prostate cancer. J Clin Oncol 2005;23:291825. http://dx.doi.org/10.1200/JCO.2005.01.529

4. Small EJ, Halabi S, Dawson NA, et al. Antiandrogen withdrawal alone or in combination with ketoconazole in androgen-independent prostate cancer patients: A phase 3 trial (CALGB 9583) J Clin Oncol 2004;22:1025-33.

http://dx.doi.org/10.1200/JCO.2004.06.037

5. Smith MR, Saad F, Chowdhury S, et al; SPARTAN Investigators. Apalutamide treatment and metastasis-free survival in prostate cancer. N Engl J Med 2018;378:140818. https://doi.org/10.1056/NEJMoa1715546

6. Hussain M, Fizazi K, Saad F, et al. Enzalutamide in men with non-metastatic, castrationresistant prostate cancer. $N$ Engl J Med 2018;378:2465-74.

https://doi.org/10.1056/NEJMoa1800536

7. Fizazi K, Shore N, Tammela TL, et al; ARAMIS Investigators. Darolutamide in nonmetastatic, castration-resistant prostate cancer. N Engl J Med 2019 Feb 14. [Epub ahead of print]. https://doi.org/10.1056/NEJMoa1815671

8. Saad F, Cella D, Basch E, et al. Effect of apalutamide on health-related quality of life in patients with non-metastatic castration-resistant prostate cancer: An analysis of the SPARTAN randomized, placebo-controlled, phase 3 trial. Lancet Oncol 2018;19:140416. https://doi.org/10.1016/S1470-2045(18)30456-X

9. Tombal B, Saad F, Penson D, et al. Patient-reported outcomes following enzalutamide or placebo in men with non-metastatic, castration-resistant prostate cancer (PROSPER): A multicentre, randomized, double-blind, phase 3 trial. Lancet Oncol 2019 Feb 12. [Epub ahead of print]. https://doi.org/10.1016/S1470-2045(18)30898-2

10. Ryan CJ, Smith MR, de Bono JS, et al. Abiraterone in metastatic prostate cancer without previous chemotherapy. N Engl J Med 2013;368:138-48.

https://doi.org/10.1056/NEJMoa1209096

11. Ryan CJ, Smith MR, Fizazi K, et al. Abiraterone acetate plus prednisone vs. placebo plus prednisone in chemotherapy-naive men with metastatic castration-resistant prostate cancer (COU-AA-302): Final overall survival analysis of a randomized, double-blind, placebo-controlled phase 3 study." Lancet Oncol 2015;16: 152-60.

https://doi.org/10.1016/S1470-2045(14)71205-7

12. de Bono JS, Logothetis CJ, Molina A, et al. Abiraterone and increased survival in metastatic prostate cancer. N Engl J Med 2011; 364:1995-2005. https://doi.org/10.1056/NEJMoa1014618 
13. Beer TM, Armstrong AJ, Rathkopf DE, et al. Enzalutamide in metastatic prostate cancer before chemotherapy. $N$ Engl J Med 2014;371:424-33.

http://dx.doi.org/10.1056/NEJMoa1405095

14. Beer TM, Armstrong AJ, Rathkopf D, et al. Enzalutamide in men with chemotherapynaive metastatic castration-resistant prostate cancer: Extended analysis of the phase 3

PREVAIL study. Eur Urol 2017;71: 151-4. https://doi.org/10.1016/j.eururo.2016.07.032

15. Scher HI, Fizazi K, Saad F, et al. Increased survival with enzalutamide in prostate cancer after chemotherapy. N Engl J Med 2012;367:1187-97. http://dx.doi.org/10.1056/NEJMoa1207506

16. Tannock IF, de Wit R, Berry WR, et al. Docetaxel plus prednisone or mitoxantrone plus prednisone for advanced prostate cancer. $N$ Engl J Med 2004;351:1502-12. http://dx.doi.org/10.1056/NEJMoa040720

17. de Bono JS, Oudard S, Ozguroglu M, et al. Prednisone plus cabazitaxel or mitoxantrone for metastatic castration-resistant prostate cancer progressing after docetaxel treatment: A randomized open-label trial. Lancet 2010;376:1147. http://dx.doi.org/10.1016/S01406736(10)61389-X

18. Eisenberger M, Hardy-Bessard AC, Kim CS, et al. Phase 3 study comparing a reduced dose of cabazitaxel $\left(20 \mathrm{mg} / \mathrm{m}^{2}\right)$ and the currently approved dose $\left(25 \mathrm{mg} / \mathrm{m}^{2}\right)$ in postdocetaxel patients with metastatic castration-resistant prostate cancer-PROSELICA. $J$ Clin Oncol 2017;35:3198-3206. https://doi.org/10.1200/JCO.2016.72.1076

19. Jankovic B, Beardsley E, Chi KN. Rechallenge with docetaxel as second-line chemotherapy in patients with metastatic hormone refractory prostate cancer (HRPC) after previous docetaxel: A population-based analysis [abstract \#196]. 2008 ASCO Genitourinary Symposium; 2008.

20. Eymard J, Oudard S, Gravis G, et al. Docetaxel reintroduction in patients with metastatic castration-resistant docetaxel-sensitive prostate cancer: A retrospective, multicentre study. BJU Int 2010;106:974-8. http://dx.doi.org/10.1111/j.1464-410X.2010.09296.x

21. Parker C, Nilsson S, Heinrich D, et al. Alpha emitter radium-223 and survival in metastatic prostate cancer. $N$ Engl J Med 2013;369:213-23. http://dx.doi.org/10.1056/NEJMoa1213755

22. Smith M, Parker C, Saad F, et al. Addition of radium-223 to abiraterone acetate and prednisone or prednisolone in patients with castration-resistant prostate cancer and bone metastases (ERA 223): A randomized, double-blind, placebo-controlled, phase 3 trial. Lancet Oncol 2019 Feb 6. [Epub ahead of print]. https://doi.org/10.1016/S14702045(18)30860-X

23. Diamond TH, Higano CS, Smith MR, et al. Osteoporosis in men with prostate carcinoma receiving androgen-deprivation therapy: Recommendations for diagnosis and therapies. Cancer 2004;100:892. http://dx.doi.org/10.1002/cncr.20056

24. Shahinian VB, Kuo YF, Freeman JL, et al. Risk of fracture after androgen deprivation for prostate cancer. $N$ Engl J Med 2005;352:154-64.

http://dx.doi.org/10.1056/NEJMoa041943

25. Smith MR, McGovern FJ, Zietman AL, et al. Pamidronate to prevent bone loss during androgen-deprivation therapy for prostate cancer. $N$ Engl J Med 2001;345:948-55. http://dx.doi.org/10.1056/NEJMoa010845 
26. Smith MR, Eastham J, Gleason DM, et al. Randomized controlled trial of zoledronic acid to prevent bone loss in men receiving androgen-deprivation therapy for non-metastatic prostate cancer. $J$ Urol 2003;169:2008-12. http://dx.doi.org/10.1097/01.ju.0000063820.94994.95

27. Smith MR, Egerdie B, Hernandez Toriz N, et al. Denosumab in men receiving androgendeprivation therapy for prostate cancer. $N$ Engl J Med 2009;361:745-55. http://dx.doi.org/10.1056/NEJMoa0809003

28. Saad F, Gleason DM, Murray R, et al. Long-term efficacy of zoledronic acid for the prevention of skeletal complications in patients with metastatic hormone refractory prostate cancer. J Natl Cancer Inst 2004;96:879-82. http://dx.doi.org/10.1093/jnci/djh141

29. Fizazi K, Carducci M, Smith M, et al. Denosumab vs. zoledronic acid for treatment of bone metastases in men with castration-resistant prostate cancer: a randomised, doubleblind study. Lancet 2011;377:813. http://dx.doi.org/10.1016/S0140-6736(10)62344-6.

30. Smith MR, Saad F, Coleman R, et al. Denosumab and bone-metastasis-free survival in men with castration-resistant prostate cancer: results of a phase 3, randomized, placebocontrolled trial. Lancet 2012;379:39-46. http://dx.doi.org/10.1016/S0140-6736(11)612269

31. Storlie JA, Buckner JC, Wiseman GA, et al. Prostate specific antigen levels and clinical response to low-dose dexamethasone for hormone-refractory metastatic prostate carcinoma. Cancer 1995;76:96-100. http://dx.doi.org/10.1002/10970142(19950701)76:1<96::AID-CNCR2820760114>3.0.CO;2-E

32. Heng DY, Chi KN. Prednisone monotherapy in asymptomatic hormone refractory prostate cancer. Can J Urol 2006;13:3335-9.

33. Loblaw DA, Mitera G, Ford M, et al. A 2011 updated systematic review and clinical practice guideline for the management of malignant extradural spinal cord compression. Int J Radiat Oncol Biol Phys 2012;84:312-7. http://dx.doi.org/10.1016/j.ijrobp.2012.01.014.

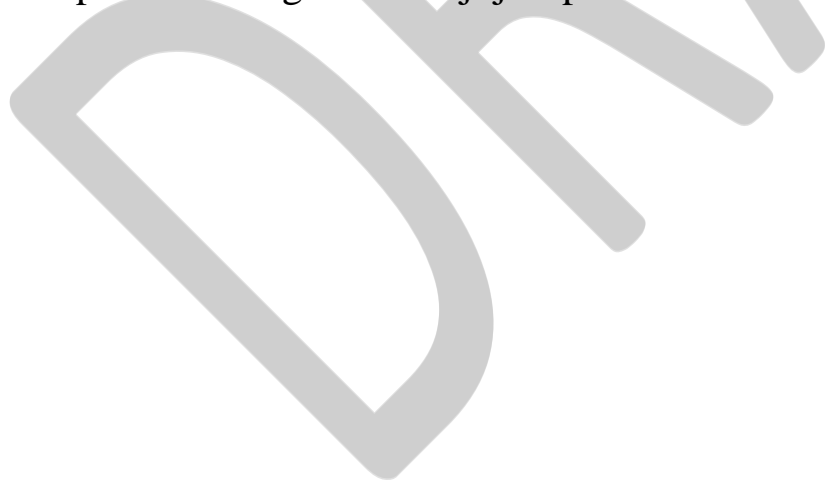




\section{Figures and Tables}

Fig. 1. Management of castration-resistant prostate cancer (CRPC). mCRPC: metastatic CRPC; PSADT: prostate-specific antigen doubling time.
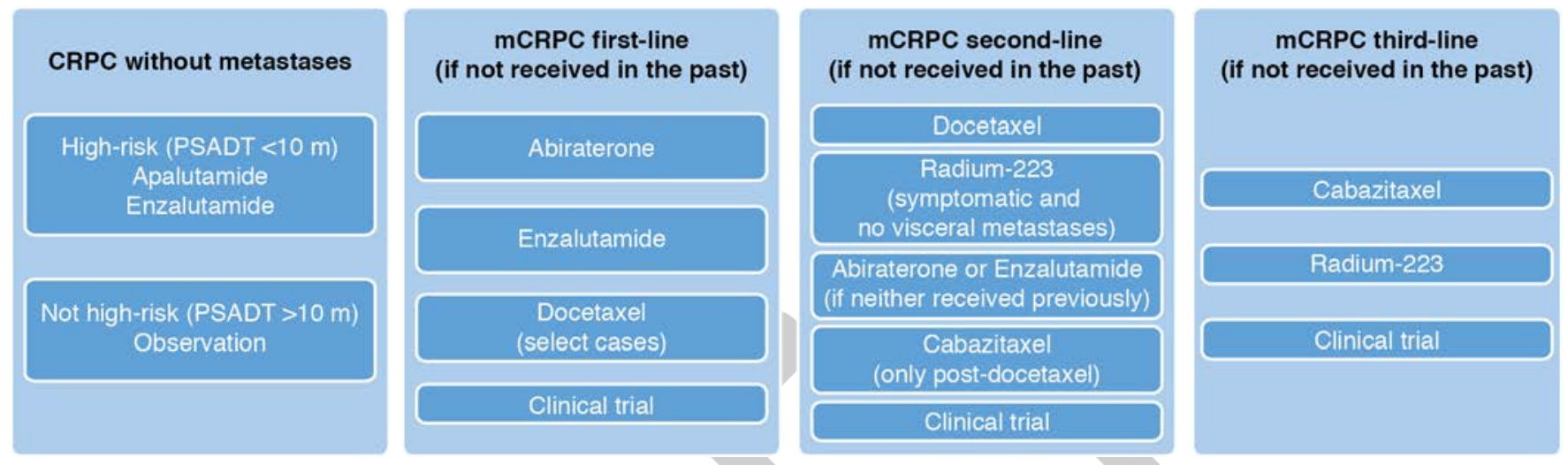

In the presence of bone metastases:

Denosumab or zoledronic acid are recommended to reduce the risk of skeletal complications

Palliative radiation therapy should be considered in patients with pain

1. The optimal sequence of available options remains unknown. In general it is felt that changing therapeutic mechanism of action with each line of therapy is likely to lead to better and longer lasting response (Expert opinion).

2. Patients who have had little no response to hormonal agents $\mathbf{O R}$ who progress with minimal change in PSA OR with significant visceral metastases should be considered for early chemotherapeutic options.

3. Radium-223 is not approved for patients with visceral metastases.

4. Whenever possible, clinical trials should remain the first choice in patients with CRPC. 


\section{CUA-CUOG guidelines summary}

Clinical scenarios and management options for patients with CRPC

Castration-resistant prostate cancer (CRPC) includes a wide range of disease types: from patients without metastases or symptoms with rising prostate-specific antigen (PSA) levels despite androgen-deprivation therapy (ADT) to patients with metastases and significant debilitation due to cancer symptoms.

Androgen-deprivation therapy and first-generation androgen receptor antagonists

Because the androgen receptor remains active in most patients who have developed castration-resistant disease, it is recommended that ADT be continued for the remainder of a patient's life (Strong Recommendation).

In patients who develop CRPC, the addition or change of first-generation androgen receptor antagonists may be considered (Level 3, Weak Recommendation).

1. Rising PSA non metastatic CRPC

1. ADT should be maintained in the nmCRPC state. First-generation androgen receptor antagonists (i.e., bicalutamide, flutamide, etc.) should be discontinued if patients are receiving these agents (Level 3, Weak Recommendation).

2. For men with high-risk nmCRPC, defined as a PSADT $<10$ months, with an estimated life expectancy of greater than five years should be offered apalutamide or enzalutamide (Level 1, Strong Recommendation).

3. In men with high-risk nmCRPC who are felt to be unsuitable or refuse approved therapies, observation or use of first-generation androgen receptor antagonists may be attempted (Level 3, Weak Recommendation).

4. Men with nmCRPC who are not considered high-risk, observation or secondary hormonal treatments may be attempted (Level 3, Weak Recommendation).

5. Patients who are untreated for nmCRPC should be followed with regular imaging every 6-12 months depending on PSADT (Level 3, Weak Recommendation).

2. Chemotherapy naïve metastatic CRPC (mCRPC) without symptoms or minimally symptomatic

Abiraterone acetate $1000 \mathrm{mg} /$ day plus prednisone $5 \mathrm{mg} /$ twice daily is recommended as first-line therapy (Level 1, Strong Recommendation).

Enzalutamide 160mg/day is recommended as first-line therapy (Level 1, Strong Recommendation). 
Docetaxel $75 \mathrm{mg} / \mathrm{m}^{2}$ every three weeks plus $5 \mathrm{mg}$ oral prednisone twice daily can be offered (Level 1, Strong Recommendation). The timing of docetaxel therapy in men with evidence of metastases but without symptoms should be discussed with the patient and therapy should be individualized based on the patient's clinical status and preference.

3. mCRPC with moderate or severe symptoms

Docetaxel $75 \mathrm{mg} / \mathrm{m}^{2}$ every three weeks plus $5 \mathrm{mg}$ oral prednisone twice daily is recommended (Level 1, Strong Recommendation).

Radium-223 every four weeks for six cycles is recommended in patients with pain due to bone metastases and who do not have visceral metastases (Level 1, Strong

Recommendation). Radium-223 significantly improved overall survival and reduced symptomatic skeletal related events in patients with symptomatic mCRPC who had previously received docetaxel chemotherapy or were deemed unfit for docetaxel.

Abiraterone acetate $1000 \mathrm{mg} /$ day plus prednisone $5 \mathrm{mg}$ twice daily or enzalutamide $160 \mathrm{mg} /$ day may be considered as first-line therapy in patients who cannot receive or refuse docetaxel (Expert opinion).

4. Metastatic CRPC who progress after docetaxel-based chemotherapy

Options with survival benefit

Cabazitaxel (25 mg/m²) plus prednisone (5 mg/day) (Level 1, Strong Recommendation)

Radium-223 every four weeks for six cycles (Level 1, Strong Recommendation)

If not received prior to docetaxel:

Abiraterone acetate (1000 mg per day) plus prednisone (5 mg twice daily) (Level 1, G

Strong Recommendation)

Enzalutamide (160 mg/day) (Level 1, Strong Recommendation)

Options with unknown survival benefit

Docetaxel plus prednisone re-exposure in patients who have had a previous favourable response to docetaxel may be reasonable (Expert opinion).

Mitoxantrone plus prednisone may be offered for palliative pain relief (Grade C).

5. Patients with CRPC and bone metastases (includes the pre- or post-chemotherapy settings) 
Denosumab (120 $\mathrm{mg}$ subcutaneous) or zoledronic acid (4 $\mathrm{mg}$ intravenous) every four weeks, along with daily calcium and vitamin $D$ supplementation, is recommended to prevent disease-related skeletal complications (Level 1, Strong Recommendation).

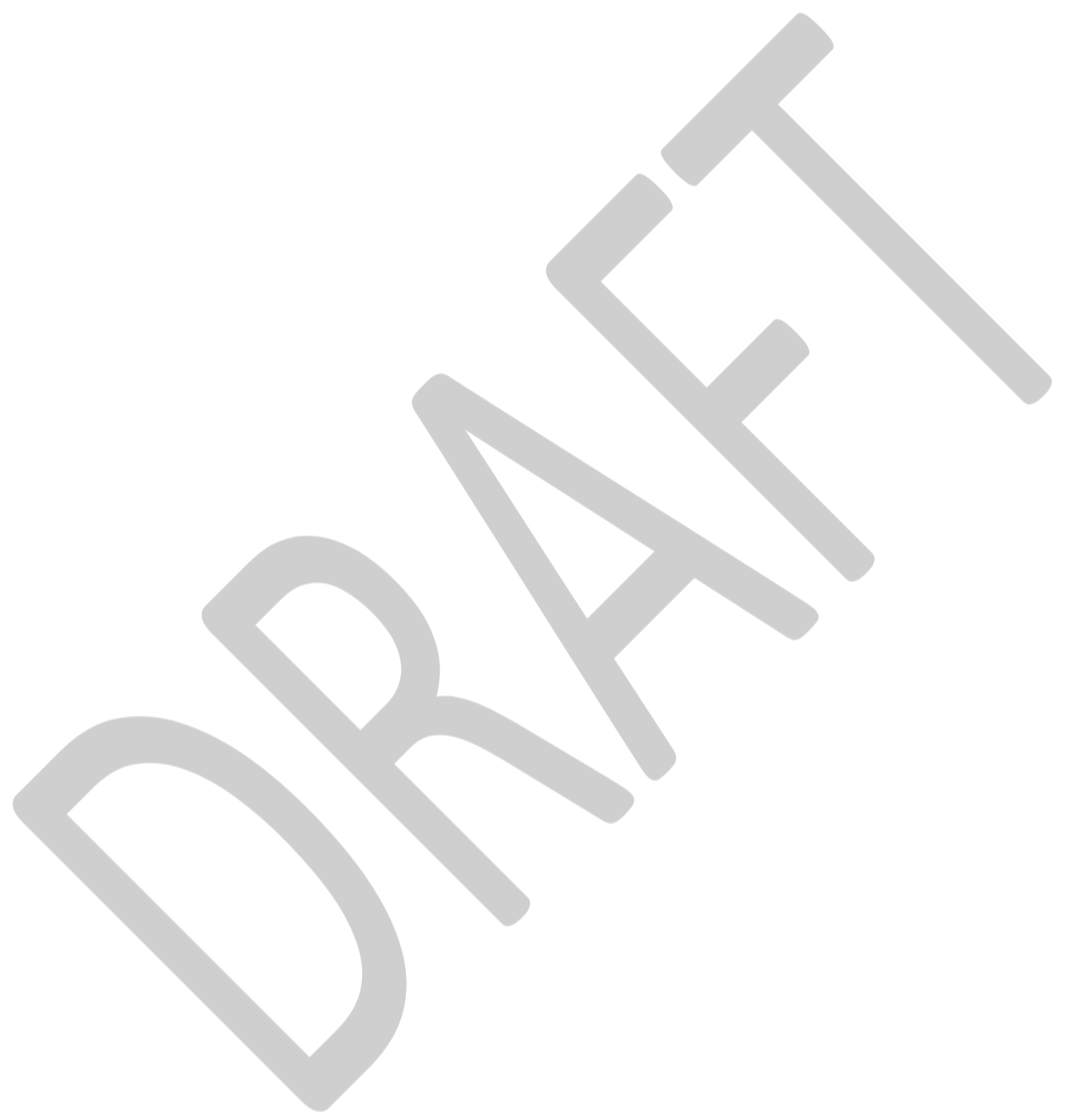

